\title{
RIPK1 is a key factor in black carbon-induced cell death
}

\author{
Xianyan $\mathrm{Xu}^{1}$, Zhaojun $\mathrm{Xu}^{2}$, Shiyong ZENG ${ }^{2}$, and Yuhui OuYANG ${ }^{3}$ \\ Departments of ${ }^{1}$ Anatomy, and ${ }^{2}$ Environmental Medicine, Quanzhou Medical College, Quanzhou, Fujian, 362011, China; ${ }^{3}$ Department \\ of Otolaryngology Head and Neck Surgery and Department of Allergy, Beijing TongRen Hospital, Affiliated with the Capital University \\ of Medical Science, Beijing, 100730, China
}

(Received 18 December 2021; and accepted 23 December 2021)

\begin{abstract}
Air pollution is associated with increased morbidity and mortality and with cell death at a cellular level. However, the exact mechanism of particulate matter-induced cell death remains to be elucidated. The aim of the present in vitro study using human alveolar epithelial cells (A549) was to determine the cell death pathway(s) induced by black carbon $(\mathrm{BC})$ and ozone oxidized-black carbon $(\mathrm{O}-\mathrm{BC})$. BC and $\mathrm{O}-\mathrm{BC}$ induced $\mathrm{A} 549$ cell death and the cytotoxic effect was dose-dependent. Cell death was significantly abrogated by inhibitor of receptor protein interacting kinase 1 (RIPK1) but only mildly inhibited by apoptosis inhibitor and RIPK3. BC- and O-BC-treated cells showed RIPK1 and RIPK3 protein overexpression and high phosphorylated levels of these proteins, as well as detectable levels of caspase- 8 active form. BC- and O-BC-triggered cell death was also fully rescued in A549 cells that under-expressed RIPK1 with RIPK1 siRNA. Our results indicated that $\mathrm{BC}$ and $\mathrm{O}-\mathrm{BC}$ could induce cell death through a multitude of pathways including apoptotic and necroptotic pathways and that RIPK1 is the upstream signal protein of these cell death pathways, with an important role in the regulation of $\mathrm{BC}$-induced cell death.
\end{abstract}

\section{INTRODUCTION}

Air pollution is a serious problem on public health. Ambient air pollutants include particles and gases, and PM2.5 (particle mass with aerodynamic diameter of $<2.5 \mu \mathrm{m})$ and tropospheric ozone have been selected as representative pollutants to quantify the health impact of ambient air pollution. Black carbon (BC) is major component of PM2.5 and is a byprod-

Address correspondence to: Zhaojun $\mathrm{Xu}, \mathrm{MD}, \mathrm{PhD}$, Department of Environmental Medicine, Quanzhou Medical College, Quanzhou, Fujian 362011, China Tel: +8659522136951

E-mail: zjxu38@hotmail.com

Yuhui Ouyang, MD, PhD, Department of Otolaryngology Head and Neck Surgery and Department of Allergy, Beijing TongRen Hospital, Affiliated with the Capital University of Medical Science, Beijing 100730, China Tel: +861058265804

E-mail: oyyuhui@sina.com uct from the incomplete burning of solid fuels, residential cooking and heating, and vehicle exhausts. The adverse health effects of $\mathrm{BC}$ exposure have been investigated in epidemiological and experimental studies; exposure to BC increases the risk of cancer, chronic obstructive pulmonary disease (COPD), and cardiovascular dysfunction (Luben et al. 2017; Niranjan and Thakur 2017).

The mechanisms of the ill-effects of $\mathrm{BC}$ include oxidative stress, breakdown of double stranded DNA and formation of DNA adducts due to reactive oxygen species (ROS)-induced enhancement of DNA damage. Exposure to $\mathrm{BC}$ is also associated with changes in DNA methylation (Rider and Carlsten 2019). As a fine particulate, $B C$ settles deep into the bronchoalveolar tree, then enters the circulation. Therefore, exposure to $\mathrm{BC}$ further induces inflammation that can lead to cell death and ultimately result in ventilatory dysfunction. BC-induced cell death is probably mediated through the apoptotic (An et al. 2019a) and autophagic pathways (An et al. 2017) 
and is even described sometimes to involve more complex molecular and cellular processes (Peixoto et al. 2017; An et al. 2019b). On the other hand, ozone, as a highly reactive irritant gas, causes oxidative stress, inflammation and lung tissue damage. Thus, the combination of ozone and PM2.5 aggravates lung damage and increases lung disease and mortality (Sunil et al. 2012).

In addition to the above mechanisms, ozone gases can cause oxidation of $\mathrm{BC}$ particles, which modifies their physicochemical characteristics, resulting in enhanced redox activity after emission into the atmosphere (Rattanavaraha et al. 2011). BC has a relatively large surface. After emission into the atmosphere, various pollutants such as heavy metals, sulfate, nitrate, polycyclic aromatic hydrocarbons (PHAs) and pathogen components adhere to the surface, synergistically increasing its toxicity. Experimentally, treatment of $\mathrm{BC}$ with ozone results in increased hydrophilicity, formulation of abundant oxygen-containing function groups and enhanced oxidative potential (Li et al. 2013; Xu et al. 2015). Several studies have assessed the differences in biological activities of $\mathrm{BC}$ and oxidized $\mathrm{BC}(\mathrm{O}-\mathrm{BC})$ (Gao et al. 2017). O-BC generates more ROS and sufficient DNA damage repair responses than BC (An et al. 2019a). O-BC exposure also induces more pro-inflammatory cellular responses than $\mathrm{BC}(\mathrm{Chu}$ et al. 2018). These results suggest that changes in particle surface properties through oxidation results in more severe genotoxicity and cytotoxicity responses than BC (Li et al. 2013; Gao et al. 2017).

The cell death processes of apoptosis and necrosis are also known as programmed and non-programmed cell death, respectively. The necrosis-like programmed cell death is currently termed necroptosis (Molnar et al. 2019). Receptor-interacting protein kinase 1 (RIPK1) is an upstream molecule on both the apoptosis and necroptosis pathways and is a key factor that regulates cell viability and inflammation (Wallach et al. 2016). It is known that TNFa binding to TNFR1 activates RIPK1, which in turn induces caspase-8-dependent apoptosis, or RIPK3 to trigger necroptosis. RIKP1 can also interact with $\mathrm{NF}-\kappa \mathrm{B}$ pathway to induce inflammatory responses and cell death. Thus, RIPK1 is associated with a wide range of cellular events spanning from inflammation to cell death. Furthermore, exposure to BC or O-BC can result in the induction of apoptosis, autophagy and necroptosis (Peixoto et al. 2017; An et al. 2019b). Although the extent of oxidative stress is considered to play an important role in determining the cell death pathway (Peixoto et al. 2017), the exact mechanism of $\mathrm{BC}$ - and $\mathrm{OBC}$-induced cell death remains obscure.

The aim of this study was to determine the cell death pathway(s) induced by $\mathrm{BC}$ and $\mathrm{O}-\mathrm{BC}$ using pharmacological approaches that included the use of specific inhibitors. The results showed that BC- and $\mathrm{O}-\mathrm{BC}$-dose-dependent induction of cell death was abrogated by RIPK1 inhibitors and partially by caspase inhibitors and RIPK3 inhibitors. The RIPK1 and RIPK3 protein and phosphorylated levels were increased in cells treated with $\mathrm{BC}$ and $\mathrm{O}-\mathrm{BC}$, and inhibition of RIPK1 expression using RIPK1 siRNA suppressed BC- and O-BC-induced cell death. Our results indicate that $\mathrm{BC}$ and $\mathrm{O}-\mathrm{BC}$ induce multiple cell death pathways.

\section{MATERIALS AND METHODS}

Reagents. IкB kinase (IKK) inhibitor BMS-345541 was purchased from Sigma Chemical Co. (St. Louis, MO); RIPK1 inhibitor Nec-1 and pan-caspase inhibitor zVAD-fmk were purchased from Selleck Chemicals (Houston, TX); and RIPK3 inhibitor GSK'872 was purchased from Calbiochem (Merck, Germany). The standard concentrations of inhibitors used in this study were selected based on previous studies: $20 \mu \mathrm{M}$ BMS-345541; $50 \mu \mathrm{M}$ Nec-1; $2 \mu \mathrm{M}$ GSK'872 and $25 \mu \mathrm{M}$ zVAD-fmk (Menon et al. 2017).

$\mathrm{BC}$ and O-BC were provided by Jing Shang (College of Environmental Sciences and Engineering, Peking University). As reported previously, Printex $\mathrm{U}$ carbon black $(\mathrm{BC})$ was purchased from Degussa, $\mathrm{O}-\mathrm{BC}$ was produced by exposure of $\mathrm{BC}$ to ozone, which was generated by photolysis of oxygen under an ultraviolet lamp (Li et al. 2013; Chu et al. 2018), and its physicochemical characteristics were similar to those described previously ( $\mathrm{Li}$ et al. 2013). Briefly, physicochemical analysis showed a particle diameter of approximately $30 \mathrm{~nm}$ for both $\mathrm{BC}$ and $\mathrm{O}-\mathrm{BC}$ with formation of larger size particles in water due to agglomeration. Treatment of the $\mathrm{BC}$ with ozone changes its surface charge from positive to negative, forms more oxygen species and free radicals on the BC surface and thus increases its oxidative capacity and cytotoxicity ( $\mathrm{Li}$ et al. 2013).

Cell culture. Human alveolar epithelial cells (A549) were obtained from the American Type Tissue Collection (Manassas, VA) (Ouyang et al. 2018). Cells were cultured in Dulbecco's Modified Eagle Medium (DMEM) (Gibco, Grand Island, NY) supplemented with $10 \%$ fetal bovine serum (FBS), 1\% penicillin/streptomycin $(10,000 \mathrm{U} / \mathrm{mL})$ at $37^{\circ} \mathrm{C}$ and 
maintained in an atmosphere of $5 \% \mathrm{CO}_{2}$.

Cell viability and inhibition assay. A549 cells were cultured in 96-well plates at a density of $5 \times 10^{4}$ cells. After 24-h culture, the cells were treated for $24 \mathrm{~h}$ with or without $\mathrm{BC}$ or ozone-BC $(\mathrm{O}-\mathrm{BC})$ at the concentration indicated in the figures. For the inhibition assay, the cells were cultured in 96-well plates at a density of $5 \times 10^{4}$ cells for $24 \mathrm{~h}$, treated with each of the inhibitors at the concentrations indicated above for $30 \mathrm{~min}$ and then stimulated with $\mathrm{BC}$ or $\mathrm{O}-\mathrm{BC}$ for $24 \mathrm{~h}$. Cell viability was assessed using Cell Counting Kit-8 (CCK-8; Dojindo Laboratory, Japan), which detects the absorbance value at $450 \mathrm{~nm}$ (OD 450) with SpectraMax 340PC384 96well plate reader (Molecular Devices).

Cell death assay by Hoechst/PI double staining. A549 cells were cultured on 8-chamber slides (Nunc International, IL) for $24 \mathrm{~h}$ and treated subsequently with $\mathrm{BC}$ or $\mathrm{O}-\mathrm{BC}$ for another $24 \mathrm{~h}$. Apoptosis and necroptosis were assessed by Hoechst and propidium iodide (PI) double staining, according to the instructions provided by the manufacturer (Solarbio, China). The cells were then examined by confocal laser scanning microscopy (Nikon A1, Tokyo).

$R N A$ interference. Silencer Pre-designed siRNAs for knockdown of RIPK1 proteins (siRNA ID \#112846, 112847,112848 ) and negative control (N.C) siRNA (siRNA ID \#462001) were purchased from Invitrogen (Carlsbad, CA). Treatment of cells with siRNA RIPK1 was performed using Lipofectamine 2000, according to the instructions provided by the manufacturer. After 24-h transfection, the cells were divided into three groups and treated with or without $\mathrm{BC}$ or $\mathrm{O}-\mathrm{BC}$ for $24 \mathrm{~h}$. Cell viability was assessed using the CCK8 assay.

Western blot. A549 cells were cultured in 6-well plates at a density of $1.5 \times 10^{5}$ cells, and after 1 day of culture, the cells were treated with $\mathrm{BC}$ or O-BC for $24 \mathrm{~h}$. The cells were lysed in a buffer containing $1 \%$ Nonidet P-40, $20 \mathrm{mM}$ Tris-HCl (pH 7.4), $150 \mathrm{mM} \mathrm{NaCl}, 1 \mathrm{mM}$ PMSF, $1 \%$ ProteinSafe Protease Inhibitor Cocktail (TransGen Biotech, Beijing, China) and $5 \mathrm{mM}$ EDTA. The protein concentrations of the lysates were detected by Pierce BCA Protein Assay (Thermo Fisher Scientific). The proteins were subjected to SDS-PAGE, and the separated protein bands were transferred to PVDF membrane (Merck Millipore, Billerica, MA) for immunoblotting using the indicated antibodies. The primary antibodies were purchased from Cell Signaling Technology (Danvers, MA). The immunoreactive bands were detected using the enhanced chemiluminescence (ECL) system (Amersham, UK) and visualized using Amersham Imager 600 (GE Healthcare, Freiburg, Germany).

\section{RESULTS}

\section{Effects of $B C$ and $O-B C$ on cell viability}

Physicochemical characterization of $\mathrm{BC}$ and $\mathrm{O}-\mathrm{BC}$ was described in Materials and Methods section. First, human alveolar epithelial cells (A549) were incubated in the presence or absence of $\mathrm{BC}$ and $\mathrm{O}-\mathrm{BC}$, and the cell viability was assessed using the CCK-8 assay. As shown in Fig. 1, both BC and $\mathrm{O}-\mathrm{BC}$ significantly and dose-dependently reduced cell viability. Treatment with $50 \mu \mathrm{g} / \mathrm{mL}$ of BC and $\mathrm{O}-\mathrm{BC}$ decreased cell viability to $23.04 \%$ and $17.26 \%$, respectively. These results suggest that both forms of $\mathrm{BC}$ are potentially cytotoxic and can induce cell death.

\section{Cell death mode}

Next, the A549 cells were incubated in the presence or absence of various cell death inhibitors to determine the cell death mode triggered by $\mathrm{BC}$ and $\mathrm{O}-\mathrm{BC}$. The IKK inhibitor BMS-345541 (BMS), a broad pan-caspase inhibitor zVAD-fmk (zVAD), RIPK1 inhibitor Nec-1 and RIPK3 inhibitor GSK'872 were added to the cell culture medium before the addition of $\mathrm{BC}$ or $\mathrm{O}-\mathrm{BC}$ treatment, followed by assessment with CCK-8 analysis. Inhibition of IKK with BMS, inhibition of apoptosis with zVAD-fmk and inhibition of necroptosis with GSK'872 resulted in only mild inhibition of cell death (Fig. 2). On the other hand, RIP1 kinase inhibitor, Nec-1, significantly reduced $\mathrm{BC}$ and O-BC-induced cytotoxicity (Fig. 2). The inhibitory effects of these inhibitors on $\mathrm{BC}$ and $\mathrm{O}-\mathrm{BC}$-induced cell death seemed comparable. These results suggest that $\mathrm{BC}$ and $\mathrm{O}-\mathrm{BC}$-induced cell death involves more than one cell death pathway. RIPK1 plays a key role in regulating the death of cells exposed to $\mathrm{BC}$ and $\mathrm{O}-\mathrm{BC}$.

\section{Complexity of $B C$ and $O-B C$-induced cell death}

We also used western blot analysis to investigate changes in the expression levels of various cell death-related proteins induced by $\mathrm{BC}$ and $\mathrm{O}-\mathrm{BC}$. Treatment of A549 cells with $\mathrm{BC}$ and O-BC upregulated the protein expression levels of RIPK1, RIPK3 and $\mathrm{I} \kappa \mathrm{B} \alpha$ proteins and their phosphorylated levels. Furthermore, $\mathrm{BC}$ and $\mathrm{O}-\mathrm{BC}$ also upregulated 


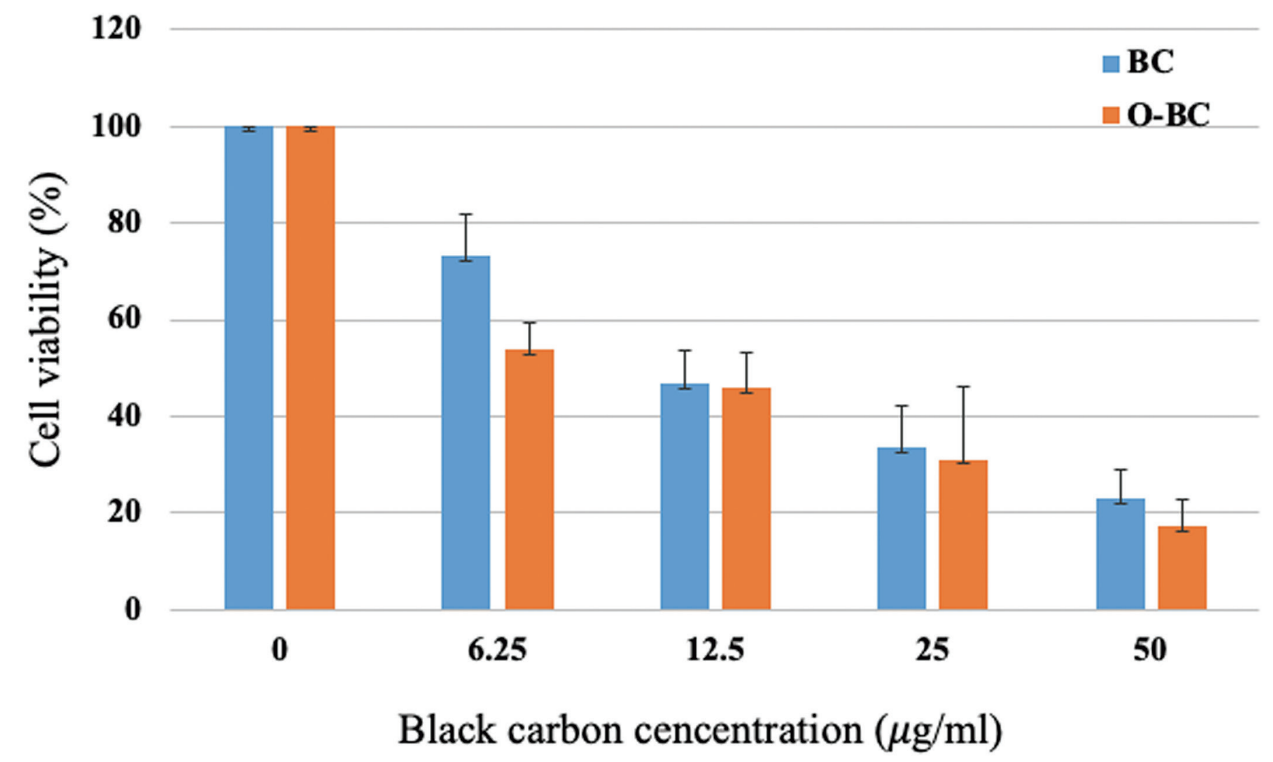

Fig. 1 Cytotoxic effect of BC and O-BC on A549 cells. The A549 cells were pre-cultured in 96 well plate, after $24 \mathrm{~h}$, cells were treated with $\mathrm{BC}$ and $\mathrm{O}-\mathrm{BC}$ in concentration as indicated. Twenty-four hours later, cell viability was subjected to CCK-8 assay. Data are mean \pm SD of at least 3 independent experiments.

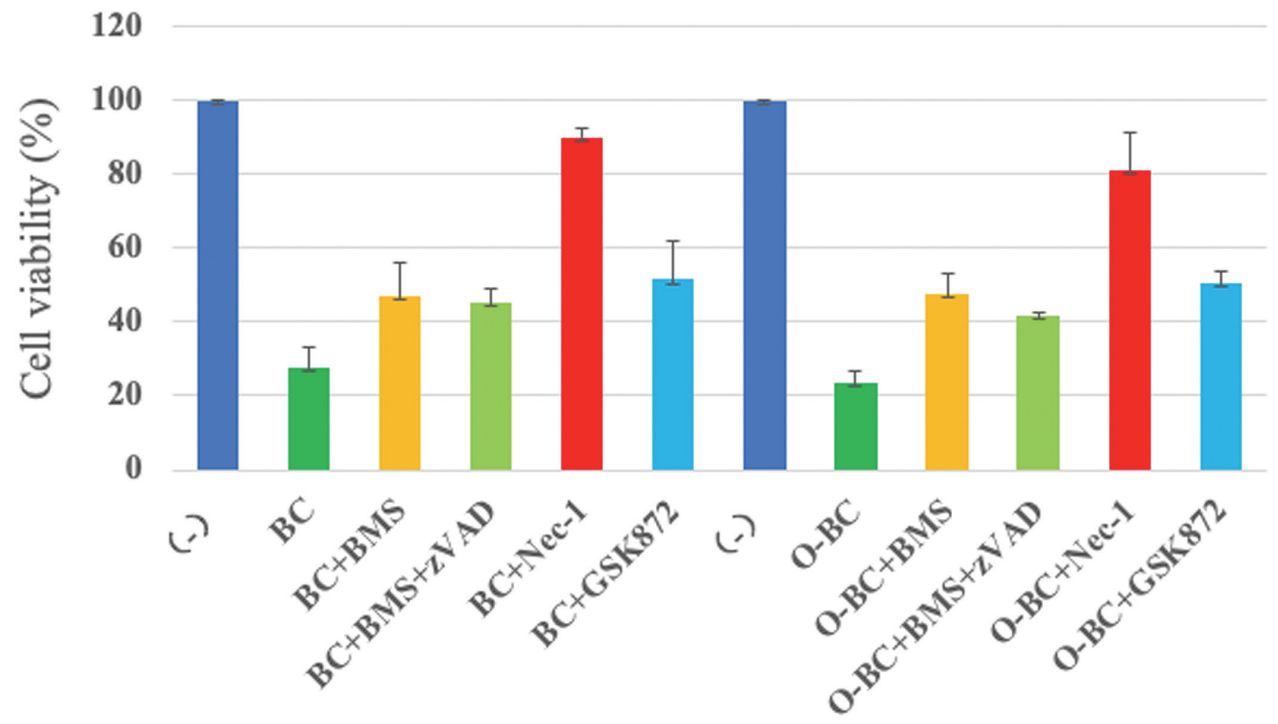

Fig. 2 Forms of cell death induced by BC and O-BC. A549 cells were pre-cultured in 96-well plate for $24 \mathrm{~h}$, and then the indicated inhibitors were added $30 \mathrm{~min}$ before the addition of $50 \mu \mathrm{g} / \mathrm{mL}$ of $\mathrm{BC}$ and $\mathrm{O}-\mathrm{BC}$. Twenty-four hours later, cell viability was assessed by CCK-8 assay. Data are mean \pm SD of at least three independent experiments.

caspase- 8 expression and enhanced the detection of the cleaved form of caspase-8 (Fig. 3A).

Exposure of A549 cells to BC and O-BC also induced apoptosis and necroptosis (Fig. 3B), which were confirmed with Hoechst/PI double staining. Staining with Hoechst/PI demonstrated cell nuclear staining with high Hoechst condensation, which is indicative of apoptosis. These results suggest that
$\mathrm{OB}$ and $\mathrm{O}-\mathrm{BC}$-induced cell death is mediated through apoptosis and necroptosis pathways as well as the NF- $\kappa \mathrm{B}$ pathway.

\section{RIPKI regulates black carbon-induced cell death} In addition to the above pharmacological approaches, we also examined the role of RIPK1 in the regulation of $\mathrm{BC}$ and $\mathrm{O}-\mathrm{BC}$ particle-induced cell death 
A

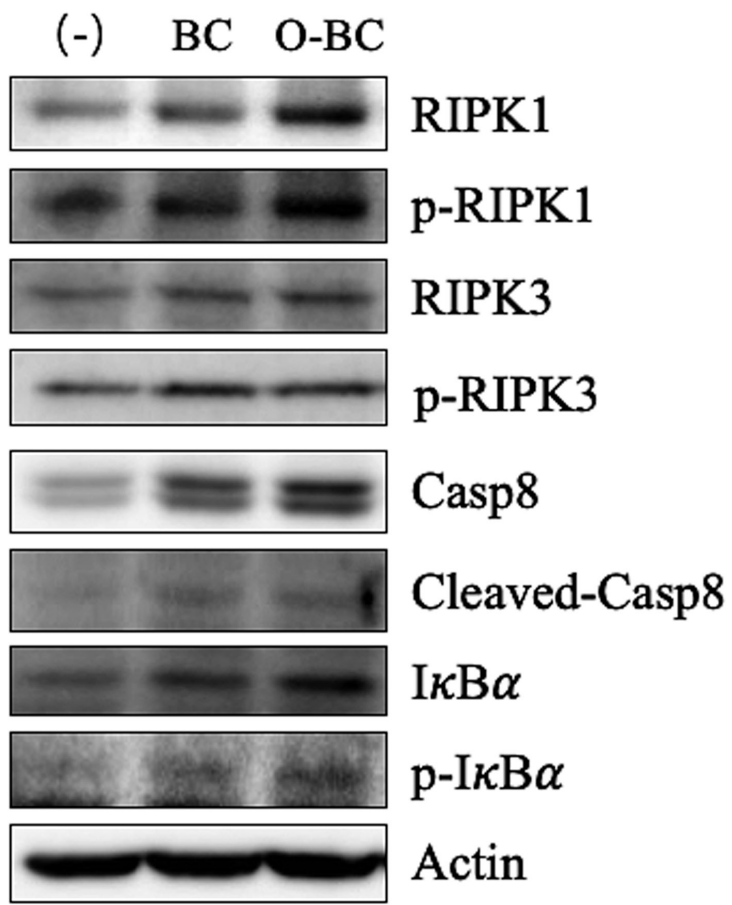

Fig. 3 Western blot analysis of apoptosis- and necroptosis-relative proteins in A549 cells treated with BC and O-BC. Proteins were extracted from cells treated for $24 \mathrm{~h}$ with $50 \mu \mathrm{g} / \mathrm{mL}$ of BC or O-BC. (A) The proteins were detected with the listed antibodies. Actin was used as a loading control.

by gene silencing technique. In these experiments, we used RIPK1 siRNA to knockdown RIPK1. We used three pre-designed independent siRNA sequences directed against human RIPK1. Western blot analysis was used to determine the knockdown efficiency of this technique. The relative RIPK1 protein level was markedly decreased in A549 cells after transfection with \#1 and \#2 si-RIPK1 (Fig. 4A). Based on these results, we used \#2 RIPK1 siRNA in the following experiment. Compared to the control siRNA, cells transfected with RIPK1-specific siRNA were completely protected against cell death induced by $\mathrm{BC}$ and O-BC. CCK-8 assay showed recovery of cell viability from $36.2 \%$ to $82.4 \%$ and $31.8 \%$ to $83.0 \%$ under RIPK1 silence conditions in A549 cells treated with $\mathrm{BC}$ and $\mathrm{O}-\mathrm{BC}$, respectively (Fig. 4B). These results are consistent with those obtained by the inhibitor assays and confirm the key role of RIPK1 in BC and O-BC-induced cell death.

\section{DISCUSSION}

Air pollution has adverse effects on human health. It is known that inhalation of PM2.5 leads to increased generation of reactive oxygen species (ROS), which can ultimately cause DNA damage, resulting inflammation and cell death. Although it has long been reported that cell death induced by $\mathrm{BC}$ and $\mathrm{O}-\mathrm{BC}$ is either apoptosis (An et al. 2019b), necrosis (An et al. 2017) and a combination of the two (Peixoto et al. 2017; An et al. 2019b), this does not reflect well the complexity of the pathology.

In the present study, first, the cell toxicity of $\mathrm{BC}$ and $\mathrm{O}-\mathrm{BC}$ was examined using the cell viability test by CCK- 8 . We found that $\mathrm{BC}$ and $\mathrm{O}-\mathrm{BC}$ resulted in cell death in a dose-dependent manner. These results are in agreement with those reported in other previous studies (Li et al. 2013; An et al. 2017). Second, we used a variety of specific inhibitors to explore the mechanism of cell death caused by $\mathrm{BC}$ and O-BC. The results showed that IKK inhibitor, apoptosis-specific pan-caspase inhibitor zVAD-fmk and necroptosis RIPK3 inhibitor partially suppressed cell death, while RIPK1 specific inhibitor Nec-1 abrogated $\mathrm{BC}$ - and $\mathrm{O}-\mathrm{BC}$-induced cell death. We also detected a cleaved caspase- 8 protein in the apoptotic active form, and up-regulation of protein expression and phosphorylation of RIPK1 and RIPK3 in BCand O-BC-treated cells. Finally, the use of RIPK1 siRNA to suppress gene expression demonstrated a limited cell death. An et al. (2019a) proposed the involvement of other forms of cell death, in addition to apoptosis, underlying $\mathrm{BC}$ cytotoxicity. Our results indicate that the cell death caused by $\mathrm{BC}$ and $\mathrm{O}-\mathrm{BC}$ is complex and exists in various forms, including apoptosis and necroptosis and are in agreement with those reported previously by other investigators, including necrosis and apoptosis in ROS-induced cell damage (Arakawa et al. 2015; Sun et al. 2018).

RIPK1 is the major modulator of cell fate under a wide range of cellular stress conditions. It is known as a molecular switch that can regulate inflammation and cell survival as well as apoptosis and necroptosis in response to various pathologic settings (Wallach et al. 2016). RIPK1 can activate the NF-KB pathway through the IKK complex to cause an inflammatory response to circumvent cell death. IKK phosphorylates the inhibitory $\mathrm{I} \kappa \mathrm{B} \alpha$ protein. As a result, $\mathrm{NF}-\kappa \mathrm{B}$ migrates to the nucleus from the cytoplasm and activates transcription of genes involved in inflammation and survival. IKK also phosphorylates and inhibits RIPK1 kinase activity to avert TNF-induced cell death (Dondelinger et al. 2015). When the NF- 
B

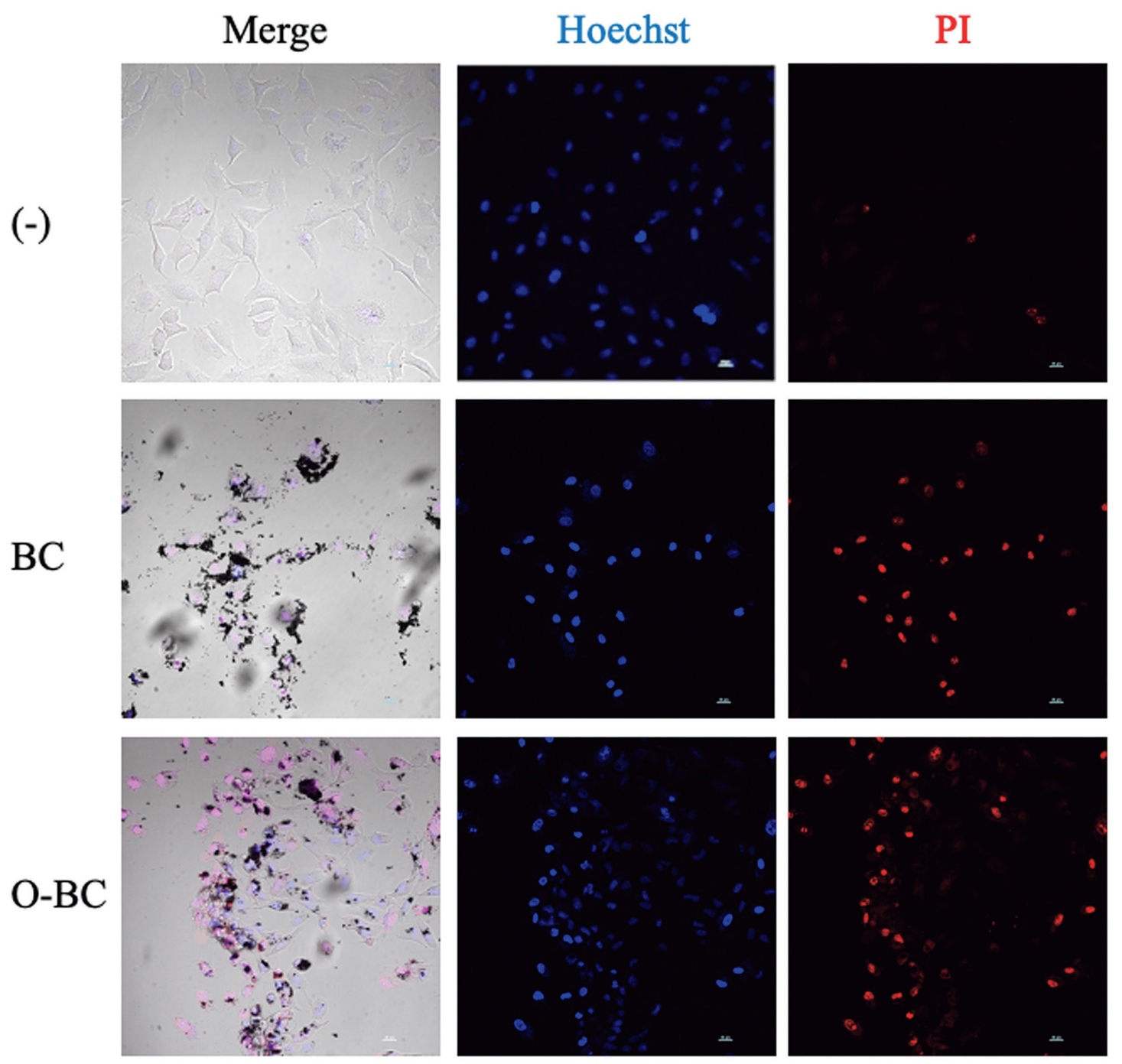

Fig. 3 (B) Cell death after 24-h treatment with $50 \mu \mathrm{g} / \mathrm{mL}$ of $\mathrm{BC}$ or O-BC was assessed by Hoechst/PI double staining and confocal microscopy. Merge: merge of transmitted detector channel, blue and red channels. Representative results of three independent experiments.

$\kappa \mathrm{B}$ signaling pathway is inhibited, RIPK1 can form a complex with Fas-associated protein with death domain (FADD) and caspase-8 (complex II) to cause apoptosis (Wegner et al. 2017). When apoptosis is inhibited through the caspase- 8 pathway, RIPK1 is phosphorylated to RIPK3 with further phosphorylation of mixed lineage kinase domain-like (MLKL), leading to the formation of a necrosome complex, with resultant switch from cell death by apoptosis to necroptosis. Our results demonstrated the induction of RIPK1 overexpression and phosphorylation by $\mathrm{BC}$ and O-BC. Both RIPK1 inhibition and expression silencing effectively promoted cell survival compared with inhibitors of apoptosis and RIPK3, indicating that RIPK1 seems to act upstream of the cell death pathway through apoptosis and necroptosis to regulate cell death. Since RIPK1 is an important regulatory factor, the signaling pathways of apoptosis and necrosis seem to be linked, though how RIPK1 precisely regulates necrosis and apoptosis is still incompletely understood (Wegner et al. 2017). We do not know which specific form of death plays a major role in BC-related cytotoxicity, and which form of cell death occurs first and occurs second, or whether they are both induced simultaneously. Further research is needed to provide answers 
A

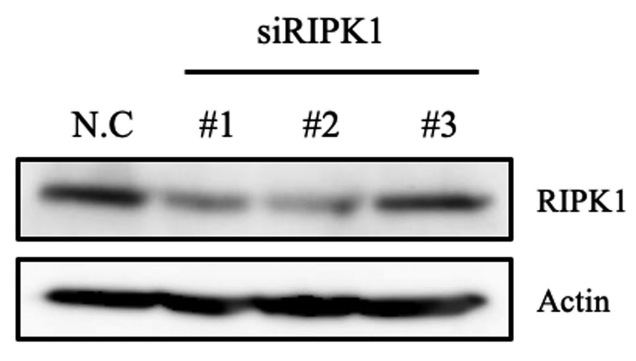

B

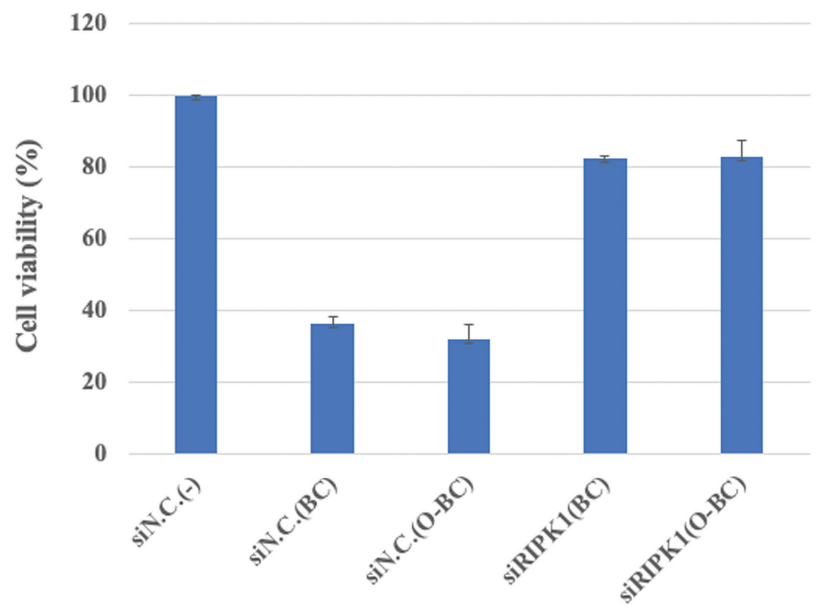

Fig. 4 Knockdown efficiency of RIPK1 siRNA and cell viability following silencing RIPK1 in A549 cells. (A) Proteins from lysates of cells transfected with RIPK1 siRNA (\#1, \#2 and \#3) or control siRNA were analyzed by western blotting using an antibody against RIPK1 or actin. (B) A549 cells were transfected with RIPK1 siRNA (\#2) for $24 \mathrm{~h}$ and treated with $50 \mu \mathrm{g} / \mathrm{mL}$ of $\mathrm{BC}$ or O-BC. Cell viability was determined $24 \mathrm{~h}$ after transfection by the CCK-8 assay. Data are mean \pm SD of at least three independent experiments.

to these issues.

Although the experimental conditions were limited to lung cell lines in this study, BC and O-BC-induced cytotoxicity, inflammation and oxidative stress at human nasal epithelial cells (hNECs) (Li et al. 2021; Ouyang et al. 2021) and human corneal epithelial cells were reported (Long et al. 2020). Further study will evaluate the possible pathological machineries with various cell-lines including macrophages and intestinal epithelial cell-lines and other immune cells.

We conclude that RIPK1 is a key molecule involved in the regulation of cell death induced by $\mathrm{BC}$ and $\mathrm{O}-\mathrm{BC}$. The results suggest that RIPK1 is a potentially useful target for the design of new treatments for diseases associated with $\mathrm{BC}$ exposure.

\section{Acknowledgments}

The project was funded by the Program for Tongjiang Scholars and Fujian Natural Science Foundation (\#2017J01152); Quanzhou City Science \& Technology Program of China (\#2018Z171, \#2018N108S), and Program for Fujian Collaborative Innovation Center (Quanzhou Medical College).

\section{CONFLICT OF INTEREST}

The authors declare no conflict of interest.

\section{REFERENCES}

An J, Zhou Q, Qian G, Wang T, Wu M, et al. (2017) Comparison of gene expression profiles induced by fresh or ozone-oxidized black carbon particles in a549 cells. Chemosphere 180, 212-220.

An J, He H, Wang L, Jin Y, Kong J, et al. (2019a) Fresh and ozonized black carbon promoted DNA damage and repair responses in a549 cells. Toxicol Res (Camb) 8, 180-187.

An J, Zhou Q, Wu M, Wang L, Zhong Y, et al. (2019b) Interactions between oxidative stress, autophagy and apoptosis in a549 cells treated with aged black carbon. Toxicol In Vitro 54, 67-74.

Arakawa S, Nakanomyo I, Kudo-Sakamoto Y, Akazawa H, Komuro I, et al. (2015) Identification of a novel compound that inhibits both mitochondria-mediated necrosis and apoptosis. Biochem Biophys Res Commun 467, 1006-1011.

Chu H, Hao W, Cheng Z, Huang Y, Wang S, et al. (2018) Black carbon particles and ozone-oxidized black carbon particles induced lung damage in mice through an interleukin-33 dependent pathway. Sci Total Environ 644, 217-228.

Dondelinger Y, Jouan-Lanhouet S, Divert T, Theatre E, Bertin J, et al. (2015) Nf-kb-independent role of $i k k \alpha / i k k \beta$ in preventing ripk1 kinase-dependent apoptotic and necroptotic cell death during tnf signaling. Mol Cell 60, 63-76.

Gao X, Xu H, Shang J, Yuan L, Zhang Y, et al. (2017) Ozonized carbon black induces mitochondrial dysfunction and DNA damage. Environ Toxicol 32, 944-955.

Li Q, Shang J and Zhu T (2013) Physicochemical characteristics and toxic effects of ozone-oxidized black carbon particles. Atmos Environ 81, 68-75. (DOI:10.1016/j.atmosenv.2013.08. 043)

Li Y, Ouyang Y, Jiao J, Xu Z and Zhang L (2021) Exposure to environmental black carbon exacerbates nasal epithelial inflammation via the reactive oxygen species (ros)-nucleo- 
tide-binding, oligomerization domain-like receptor family, pyrin domain containing 3 (nlrp3)-caspase-1-interleukin $1 \beta$ (il-1 $\beta$ ) pathway. Int Forum Allergy Rhinol 11, 773-783.

Long Q, Huang Y, Shang J, Liu Y and Chen C (2020) Black carbon induces cytotoxicity and nlrp3 inflammasome activation in human corneal epithelial cells. Curr Eye Res 45, 680 685.

Luben TJ, Nichols JL, Dutton SJ, Kirrane E, Owens EO, et al. (2017) A systematic review of cardiovascular emergency department visits, hospital admissions and mortality associated with ambient black carbon. Environ Int 107, 154-162.

Menon MB, Gropengiesser J, Fischer J, Novikova L, Deuretzbacher A, et al. (2017) P38(mapk)/mk2-dependent phosphorylation controls cytotoxic ripk1 signalling in inflammation and infection. Nat Cell Biol 19, 1248-1259.

Molnar T, Mazlo A, Tslaf V, Szollosi AG, Emri G, et al. (2019) Current translational potential and underlying molecular mechanisms of necroptosis. Cell Death Dis 10, 860.

Niranjan R and Thakur AK (2017) The toxicological mechanisms of environmental soot (black carbon) and carbon black: Focus on oxidative stress and inflammatory pathways. Front Immunol 8, 763.

Ouyang Y, Li Y, Xu Z, An Y and Zhang L (2021) Assessment of changes in genetic transcriptome in nasal epithelial cells exposed to ozone-aged black carbon and pollen allergen by high-throughput transcriptomics. Allergy Asthma Clin Immunol 17, 52.

Ouyang Y, Xu Z, Fan E, Li Y, Miyake K, et al. (2018) Changes in gene expression in chronic allergy mouse model exposed to natural environmental pm2.5-rich ambient air pollution. Sci Rep 8, 6326.

Peixoto MS, de Oliveira Galvao MF and Batistuzzo de Medeiros SR (2017) Cell death pathways of particulate matter toxicity. Chemosphere 188, 32-48.

Rattanavaraha W, Rosen E, Zhang H, Li Q, Pantong K, et al. (2011) The reactive oxidant potential of different types of aged atmospheric particles: An outdoor chamber study. Atmos Environ 45, 3848-3855. (DOI:10.1016/j.atmosenv.2011. 04.002)

Rider CF and Carlsten C (2019) Air pollution and DNA methylation: Effects of exposure in humans. Clin Epigenetics 11, 131.

Sun Y, Wang C, Wang L, Dai Z and Yang K (2018) Arsenic trioxide induces apoptosis and the formation of reactive oxygen species in rat glioma cells. Cell Mol Biol Lett 23, 13.

Sunil VR, Patel-Vayas K, Shen J, Laskin JD and Laskin DL (2012) Classical and alternative macrophage activation in the lung following ozone-induced oxidative stress. Toxicol Appl Pharmacol 263, 195-202.

Wallach D, Kang TB, Dillon CP and Green DR (2016) Programmed necrosis in inflammation: Toward identification of the effector molecules. Science 352, aaf2154.

Wegner KW, Saleh D and Degterev A (2017) Complex pathologic roles of ripk1 and ripk3: Moving beyond necroptosis. Trends Pharmacol Sci 38, 202-225.

Xu W, Li Q, Shang J, Liu J, Feng X, et al. (2015) Heterogeneous oxidation of so2 by o3-aged black carbon and its dithiothreitol oxidative potential. J Environ Sci (China) 36, 56-62. 